\title{
Aplicação de Multiplicadores de Lagrange em economia
}

\section{Bianca Masques Prescott* $\quad$ Carlos Frederico F. B. Vasconcellos ${ }^{1}$}

\author{
Instituto de Matemática e Estatística-UERJ \\ R. São Francisco Xavier, 524 bloco C - CEP 20550-013, Rio de Janeiro, RJ, Brasil. \\ E-mails: biancaprescott@hotmail.com cfredvasc@ime.uerj.br
}

\section{$\underline{\text { Resumo }}$}

Este trabalho tem como objetivo mostrar a aplicação do Multiplicador de Lagrange para resolver problemas de otimização em economia, devido ao fato de que estas questões geram importantes sugestões e conclusões sobre o comportamento econômico do consumidor.

Diversas questões de interesse econômico e processos de otimização estão modelados através de extremos condicionados, e algumas questões típicas na formulação dessas questões são as seguintes:

i. As funções nem sempre são dadas explicitamente, então se considera que elas tenham propriedades qualitativas características, assim os problemas têm um caráter mais "teórico" que "computacional";

ii. Normalmente, o economista não está interessado em encontrar o valor ótimo restrito, mas sim nas consequências da otimização, sendo assim, o problema não é "encontrar o mínimo", mas "assumindo que o mínimo é alcançado, quais consequências podem ser deduzidas?”;

iii. O Multiplicador de Lagrange $\lambda$, geralmente, pode ser interpretado como a taxa de variação do valor ótimo em relação a algum parâmetro;

iv. O teorema da função implícita desempenha papel crucial no estudo do problema, uma vez que a análise requer a solução de um sistema de equações lineares para as variáveis endógenas (dependentes) e computar as derivadas parciais destas variáveis em relação as variáveis exógenas (independentes).

Para iniciar o desenvolvimento do trabalho foram considerados alguns resultados preliminares: Utilidade Marginal, Produtividade Marginal, Estática Comparativa, Cálculo da Matriz inversa por Cofator, Teorema da Função Implícita, Teoremas relacionados ao Multiplicador de Lagrange e Condição suficiente para um ponto crítico ser extremo local.

Foram apresentados alguns exemplos clássicos da utilização do Multiplicador de Lagrange em problemas matemáticos e econômicos, no intuito de ter uma visão geral e

\footnotetext{
${ }^{1}$ Professor do Departamento de Análise Matemática IME-UERJ

*Aluna de Licenciatura em Matemática IME-UERJ e aluna de IC
} 
ampliada das diferentes formas de aplicação do Multiplicador de Lagrange em problemas de otimização. Estes exemplos tratam dos seguintes pontos:

- Minimização de funções sujeitas a vínculos;

- Pontos de máximo e mínimo de funções sujeitas a vínculos;

- Maximização de volume;

- Problemas econômicos onde são dadas:

- Uma função que define a quantidade de espaço requerida por uma empresa, o custo da mão de obra e do capital, sendo pedido para que se determinem as quantidades de capital e mão de obra que devem ser utilizadas de forma que o espaço seja o mínimo possível.

○ Uma função da produção de uma firma, o custo do capital, da mão de obra e a quantidade de bens a serem produzidos, sendo pedido:

a. As quantidades de mão-de-obra e capital que devem ser utilizados para minimizar o custo;

b. O valor do M.L. no nível ótimo de produção;

c. Mostrar que no nível de produção ótimo existe a seguinte relação:

$\frac{[\text { Produtividade marginal de mão de obra }]}{[\text { Produtividade marginal de capital }]}=\frac{[\text { Preço da unidade de mão de obra }]}{[\text { Preço da unidade de capital }]}$

A principal aplicação do Multiplicador de Lagrange em economia foi abordada a partir do seguinte exemplo ilustrativo:

- Assumindo a utilidade obtida a partir do consumo de dois produtos $x$ e $y$, que são adquiridos no mercado em quantidades $X$ e $Y$, respectivamente, e de certa quantidade $L$ de tempo de lazer $l$. A teoria da escolha do consumidor pode ser caracterizada matematicamente como um problema de otimização restrito em que o indivíduo "escolhe", a quantidade de lazer $l$ (e implicitamente rendimento de dinheiro) e as quantidades de $x$ e $y$ consumidas. Sejam $\mathrm{P}_{\mathrm{x}}$ e $\mathrm{P}_{\mathrm{y}}$ o preço por unidade de $x$ e $y$, respectivamente, $w$ a taxa de salário, $T$ o tempo total disponível e $L$ a quantidade de tempo de lazer escolhido pelo indivíduo por período (por exemplo, por semana, mês ou ano). O tempo de trabalho então pode ser definido como $T-L$. Admitindo que toda renda será gasta, a restrição orçamentária passa a ser: $w(T-L)-P_{x} X-P_{y} Y=0$

Para o economista a questão central é analisar o impacto sobre as variáveis endógenas de alterações nas variáveis exógenas, assumindo que o indivíduo responde a 
tais alterações fazendo um conjunto diferente de escolhas, a fim de maximizar a utilidade no contexto alterado.

Assim, foi realizada uma análise do exemplo ilustrativo para se verificar alguns conceitos relacionados à economia, sendo esses conceitos apresentados ao longo do desenvolvimento de diversos cálculos e suposições sobre as variáveis.

Todo o trabalho foi direcionado para que pudesse ser verificado as consequências da otimização, que como foi dito inicialmente, são de interesse aos economistas.

O multiplicador de Lagrange pode ser interpretado como a utilidade marginal da taxa de salário por hora de trabalho e pode-se demonstrar que é proporcional à utilidade marginal da renda.

Uma consequência importante da análise feita é que um corte no imposto de renda projetado para estimular o esforço de trabalho, elevando as taxas de imposto salarial (economia do lado da oferta), pode gerar pouca resposta, porque esse corte dará origem tanto a efeitos de substituição quanto a efeitos na renda.

A abordagem ilustrada no trabalho, através desse último exemplo, é útil no tratamento de uma variedade de questões em economia. Diversos outros tipos de problemas, podem ser explorados pelo leitor ou estudante interessado em aplicações de matemática em Economia, que servem para aplicar o método "Multiplicador de Lagrange" e verificar quais características do problema estudado reaparecem, talvez com alguma modificação.

Palavras-chave: Multiplicadores de Lagrange, Otimização, Economia Matemática

\section{Referências}

[1] D. Pinto e Maria C. Ferreira Morgado, "Calculo Diferencial e Integral de funções de varias variáveis", Editora UFRJ,2004.

[2] Larry J. Goldstein, David C. Lay e David I. Schneider, "Matemática Aplicada", Editora Bookman,2000.

[3] R. E. Williamson, R. H. Crowell e H. F. Trotter, "Calculus of Vector Functions", Third Edition, Prentice Hall, 1972.

[4] W. Rudin, "Princípios de Análise Matemática”, Editora Universidade de Brasília, 1976.

[5] John V. Baxley and John C. Moorhouse, Lagrange Multiplier Problems in Economics, The American Mathematical Monthly, Vol. 91, p. 404-412, 1984.

*Aluna de Licenciatura em Matemática IME-UERJ e aluna de IC 